\title{
A Novel RF Sensing Circuit Using Injection Locking and Frequency Demodulation for Cognitive Radio Applications
}

\author{
Chien-Jung Li, Student Member, IEEE, Fu-Kang Wang, \\ Tzyy-Sheng Horng, Senior Member, IEEE, and Kang-Chun Peng, Member, IEEE
}

\begin{abstract}
A novel RF sensing circuit for a cognitive radio to sense spectral environment is proposed using injection locking and frequency demodulation techniques. The research starts with deriving a generalized locking equation for oscillators as a theoretical basis, and then develops the sensing principle and circuit architecture to a practical and useful level. To accurately evaluate the sensing performance, a discrete time approach is investigated to compute the sensed output signal. In the experiments, a prototype circuit is designed in the $2.4-\mathbf{G H z}$ industrial-scientific-medical band to cover a sensing frequency range from 2.4 to $2.484 \mathrm{GHz}$. The spectrum scanning over the 84-MHz bandwidth is accomplished in less than $1 \mathrm{~ms}$. The experimental results agree quite well with the theoretical predictions, showing that the proposed RF sensing circuit can fast and reliably detect frequency and power for analog and digital modulation signals.
\end{abstract}

Index Terms-Cognitive radio, frequency demodulation, injection locking, RF sensing circuit, spectrum scanning, spectrum sensing.

\section{INTRODUCTION}

$\mathbf{T}$ HE efficiency of spectral utilization has been brought to light by the Federal Communications Commission (FCC) in the past few years. The FCC reported that the maximum utilization of the allocated spectrum was ranging from $15 \%$ to $85 \%$ [1], and the average utilization of the licensed television broadcast spectrum was only $14 \%$ [2]. On the basis of these observations, the FCC recently proposed the legal operation of unlicensed devices that can coexist with primary users in white spaces to significantly increase the spectral efficiency. A cognitive radio technology, which is an extension of software-defined radio (SDR), is one of the solutions that can accomplish the coexistence mechanism [3], [4]. Characteristics such as wideband capability of spectrum sensing, spectrum sharing, and frequency agility have become the dominant design criteria by

Manuscript received April 15, 2009; revised September 18, 2009. First published November 03, 2009; current version published December 09, 2009. This work was supported in part by the National Science Council, Taiwan, under Grant 97-2221-E-110-035-MY3 and Grant 97-2622-E-110-007-CC1, and by the Department of Industrial Technology, Taiwan, under Grant 97-EC-17-A-01-S1-104.

C.-J. Li, F.-K. Wang, and T.-S. Horng are with the Department of Electrical Engineering, National Sun Yat-Sen University, Kaohsiung 804, Taiwan (e-mail: m9131624@student.nsysu.edu.tw; m963010016@student.nsysu.edu.tw; jason@ee.nsysu.edu.tw).

K.-C. Peng is with the Department of Computer and Communication Engineering, National Kaohsiung First University of Science and Technology, Kaohsiung 811, Taiwan (e-mail: peterpkg@ccms.nkfust.edu.tw).

Digital Object Identifier 10.1109/TMTT.2009.2034415 which cognitive radio systems are judged. Motivated by the above goals, a cognitive radio generally requires a stringent frequency synthesizer with short enough settling time for fast frequency switching speed [5]. Recently, Ackland [6] proposed a tri-band cognitive radio front-end using a spectrum monitoring receiver, which down-converts the RF signal to the IF and applies the power detection technique to sense the spectral environment. This kind of receiver needs to be based on a high-speed frequency synthesizer, which increases the system complexity and hence the power consumption and the cost.

In this paper, a simpler RF sensing circuit architecture is employed without using the frequency synthesizer. The proposed technique mainly uses injection locking and frequency demodulation of a voltage-controlled oscillator (VCO) to achieve a high sensing speed and a stable sensing operation. The preliminary publication of this work [7] provided brief theoretical presentations and case studies for sensing sinusoidal and constant-envelope modulated signals. This paper substantially expands [7] to include detailed theory and new application to sense the non-constant envelope modulated signal. In addition, this paper extends the sensing circuit function to support additional receiver detection.

The remainder of this paper is organized as follows. Section II begins with deriving the locking equation for an oscillator under the influence of modulation signal injections. The background information related to injection locking is also reviewed. Sections III and IV respectively presents the proposed RF sensing circuit architecture, and provides the discrete time analysis to predict the sensing behavior. Section V discusses the computed and experimental results. Finally, Section VI summarizes and concludes this work.

\section{GeNERALIZED LOCKING EQUATION AND CHARACTERISTICS FOR OSCILLATORS}

\section{A. Review and Proposed Approach}

The synchronization of an oscillator under the influence of injecting a sinusoidal signal has been studied by Adler [8] and many other authors [9]-[13]. Adler used a vector diagram to describe the relation between an oscillator output signal and an injection signal; he derived a phase differential equation that accounts for many of the observed phenomena of synchronization by assuming that a weak injection signal is applied to a free-running oscillator. In 1947, Huntoon and Weiss [9] developed a 


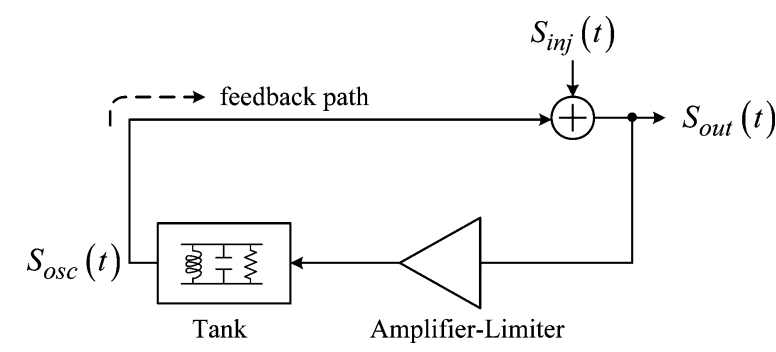

Fig. 1. Block diagram of an oscillator under injection.

synchronization equation similar to Adler's equation by considering a fictitious change of load impedance subject to injections. In 1965, Adler's work was extended by Paciorek [10] to consider higher levels of injection signals. In the last few years, Razavi [12] and Mirzaei [13], respectively, derived a locking equation, which is identical to that of Adler's and Paciorek's results, by using a different approach. Although a great deal of effort has been made on the sinusoidal signal injection, what seems to be lacking, however, is deriving a locking equation related to the modulated injection signals. Based on Adler's approach, we are concerned with the development of a detailed model, which is suitable for modulation signal injections. Furthermore, this model will be used to characterize the RF sensing circuit in the later sections.

Fig. 1 displays the block diagram of an oscillator, which consists of an amplifier-limiter, a tank circuit, and a feedback path. The amplifier-limiter is an amplifier with a nonlinear element to stabilize the amplitude of a self-excited oscillator. The tank circuit has a resonant frequency to determine the frequency of a free-running oscillator. The feedback path forms a loop to establish the oscillation condition. It is noted that the feedback path includes a summing point to allow the injection of signals. In this figure, $S_{\text {osc }}(t)$ represents the inherent oscillation signal, $S_{\text {inj }}(t)$ represents the injection signal, and $S_{\text {out }}(t)$ is the resultant oscillator output signal under injection.

Let us begin by considering the condition when no external signal is injected to an oscillator free-running at a frequency determined by the tank circuit. In this condition, $S_{\text {out }}(t)$ and feedback signal $S_{\text {osc }}(t)$ must coincide for the oscillator to maintain oscillation at the resonant frequency of the tank circuit. Next, let us turn to a consideration of the condition when a sinusoidal signal of slightly different frequency is injected to the oscillator. As shown in Fig. 1, the feedback signal $S_{\text {osc }}(t)$ combines with the injection signal $S_{\mathrm{inj}}(t)$ to output $S_{\text {out }}(t)$. The addition of $S_{\text {inj }}(t)$ induces a phase shift between $S_{\text {osc }}(t)$ and $S_{\text {out }}(t)$; this forces the free-running oscillator to change to a new oscillation frequency for the tank circuit to introduce a necessary phase shift to satisfy the new oscillation condition.

Fig. 2 illustrates the vector representation of the signals shown in Fig. 1. To begin with, we first consider that the modulated injection signal $S_{\mathrm{inj}}(t)$ has a time-varying amplitude $E_{\text {inj }}(t)$ and an instantaneous frequency $\omega_{\mathrm{inj}}(t)$, and $S_{\mathrm{inj}}(t)$ is at rest at any instant with respect to our eyes. The instantaneous frequency of the injection signal can be written as

$$
\omega_{\text {inj }}(t)=\omega_{\text {inj }}+\frac{d \theta_{i}(t)}{d t}
$$

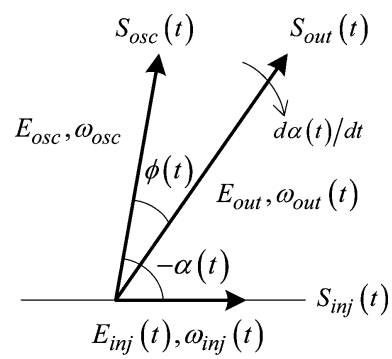

Fig. 2. Vector diagram of the instantaneous voltages used in Fig. 1.

where $\omega_{\mathrm{inj}}$ is the center frequency of $S_{\mathrm{inj}}(t)$ and $d \theta_{i}(t) / d t$ expresses the instantaneous modulation frequency of $S_{\mathrm{inj}}(t)$. The inherent oscillation signal $S_{\text {osc }}(t)$ has a constant amplitude $E_{\text {osc }}$ in nature and the oscillator is free-running at frequency $\omega_{\text {osc }}$. The resultant oscillator output signal $S_{\text {out }}(t)$ is also considered to have a constant amplitude $E_{\text {out }}$ and an instantaneous output frequency $\omega_{\text {out }}(t)$. As depicted in Fig. 2, $S_{\text {out }}(t)$ can also be regarded as a vector rotating clockwise with a beat frequency $d \alpha(t) / d t$ with respect to $S_{\mathrm{inj}}(t)$ so that the instantaneous frequency shall be represented as

$$
\omega_{\text {out }}(t)=\omega_{\text {inj }}(t)+\frac{d \alpha(t)}{d t}
$$

From an inspection of the vector diagram in Fig. 2, at any instant, we can derive the following relations:

$$
\begin{aligned}
& E_{\text {out }} \sin \phi(t)=E_{\text {inj }}(t) \sin (-\alpha(t)) \\
& E_{\text {out }} \cos \phi(t)=E_{\text {osc }}+E_{\text {inj }}(t) \cos (-\alpha(t))
\end{aligned}
$$

where the phase difference $\phi(t)$ between $S_{\text {osc }}(t)$ and $S_{\text {out }}(t)$ is resulted from the addition of $S_{\mathrm{inj}}(t)$. Dividing (3) by (4) yields

$$
\tan \phi(t)=-\frac{E_{\text {inj }}(t) \sin \alpha(t)}{E_{\text {osc }}+E_{\text {inj }}(t) \cos \alpha(t)} .
$$

Replacing (1) and (2) into the phase shift for a tank circuit given in [14] leads to

$$
\begin{aligned}
\tan \phi(t) & =\frac{2 Q}{\omega_{\text {osc }}}\left[\omega_{\text {out }}(t)-\omega_{\text {osc }}\right] \\
& =\frac{2 Q}{\omega_{\text {osc }}}\left\{\frac{d \alpha(t)}{d t}-\left[\omega_{\text {osc }}-\omega_{\text {inj }}-\frac{d \theta_{i}(t)}{d t}\right]\right\}
\end{aligned}
$$

where $Q$ is the quality factor of the tank circuit. Proceeding with the derivation, making (5) equal to (6) results in

$$
\frac{d \alpha(t)}{d t}=-B(t) \sin \alpha(t)+\Delta \omega_{\mathrm{osc}}-\frac{d \theta_{i}(t)}{d t}
$$

where

$$
B(t)=\frac{\omega_{\mathrm{osc}}}{2 Q} \frac{E_{\mathrm{inj}}(t)}{E_{\mathrm{osc}}} \frac{1}{1+\frac{E_{\mathrm{inj}}(t)}{E_{\mathrm{osc}}} \cos \alpha(t)}
$$

and

$$
\Delta \omega_{\mathrm{osc}}=\omega_{\mathrm{osc}}-\omega_{\mathrm{inj}}
$$

is the undisturbed beat frequency that defines the spontaneous frequency separation between the oscillator inherent frequency and the injection frequency. In contrast to [8] and [9], the beat 
frequency $d \alpha(t) / d t$ evidently relates to the instantaneous modulation frequency $d \theta_{i}(t) / d t$ and injection amplitude $E_{\text {inj }}(t)$. Equation (7) is the generalized locking equation describing the phase dynamics of an oscillator under the influence of a modulation injection signal with time-varying envelope and phase components.

Consider that the injection signal is sinusoidal with constant amplitude and frequency, i.e., $E_{\text {inj }}(t)=E_{\text {inj }}$ and $d \theta_{i}(t) / d t=$ 0 , (7) becomes

$$
\frac{d \alpha(t)}{d t}=-\frac{\omega_{\mathrm{osc}}}{2 Q} \frac{E_{\mathrm{inj}}}{E_{\mathrm{osc}}} \frac{\sin \alpha(t)}{1+\frac{E_{\mathrm{inj}}}{E_{\mathrm{osc}}} \cos \alpha(t)}+\Delta \omega_{\mathrm{osc}}
$$

which is identical to that of Paciorek's result [10]. If we substitute a current ratio $I_{\mathrm{inj}} / I_{\mathrm{osc}}$ for the voltage ratio $E_{\mathrm{inj}} / E_{\text {osc }}$ into (10), we will find the same result with the generalized Adler's equation derived by Mirzaei [13]. Furthermore, consider that the injection signal is sinusoidal and the amplitude level is weak. In this condition, (10) can be simplified to the well-known Adler's equation [8]

$$
\frac{d \alpha(t)}{d t}=-\frac{\omega_{\mathrm{osc}}}{2 Q} \frac{E_{\mathrm{inj}}}{E_{\mathrm{osc}}} \sin \alpha(t)+\Delta \omega_{\mathrm{osc}} .
$$

\section{B. Locking Range}

The locking range $\omega_{\mathrm{LR}}$ defines the maximum undisturbed beat frequency for the oscillator to be locked with a sinusoidal injection signal. When locking occurs, the phase difference $\alpha(t)$ goes toward a constant value as time increases. This constant value can be denoted as $\alpha_{\infty}$ to express the phase difference between $S_{\text {osc }}(t)$ and $S_{\text {inj }}(t)$ in the steady state, and found by making (10) approach zero to obtain

$$
\Delta \omega_{\mathrm{osc}} \approx \frac{\omega_{\mathrm{osc}}}{2 Q} \frac{E_{\mathrm{inj}}}{E_{\mathrm{osc}}} \frac{\sin \alpha_{\infty}}{1+\frac{E_{\mathrm{inj}}}{E_{\mathrm{osc}}} \cos \alpha_{\infty}} .
$$

By differentiating $\Delta \omega_{\text {osc }}$ with respect to $\alpha_{\infty}$, the maximum undisturbed beat frequency takes place when $\cos \alpha_{\infty}=-E_{\text {inj }} / E_{\text {osc }}$. Substituting this condition back into (12) leads to the expression of locking range given as [10]

$$
\omega_{\mathrm{LR}}=\frac{\omega_{\mathrm{osc}}}{2 Q} \frac{E_{\mathrm{inj}}}{E_{\mathrm{osc}}} \frac{1}{\sqrt{1-\left(\frac{E_{\mathrm{inj}}}{E_{\mathrm{osc}}}\right)^{2}}} .
$$

When the injection level is weak, (13) can then be simplified as [8]

$$
\omega_{\mathrm{LR}} \approx \frac{\omega_{\mathrm{osc}}}{2 Q} \frac{E_{\mathrm{inj}}}{E_{\mathrm{osc}}}
$$

Note that $\omega_{\text {LR }}$ is approximately proportional to the voltage amplitude ratio $E_{\text {inj }} / E_{\text {osc }}$ or the square root of power ratio $\sqrt{P_{\text {inj }} / P_{\text {osc }}}$, where $P_{\text {inj }}$ and $P_{\text {osc }}$ are the inherent oscillation output power and the injection signal power, respectively. When $\Delta \omega_{\text {osc }}$ is smaller than $\omega_{\mathrm{LR}}$, the pull-in process [8] will pull the oscillator output frequency $\omega_{\text {out }}(t)$ to lock on the injection frequency $\omega_{\text {inj. }}$. In other words, the oscillator is under the injection-locking condition.
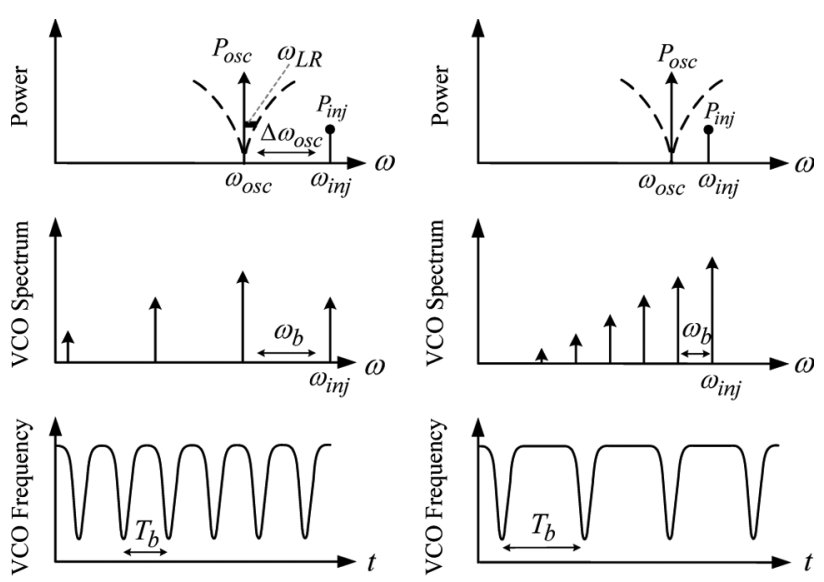

(a)

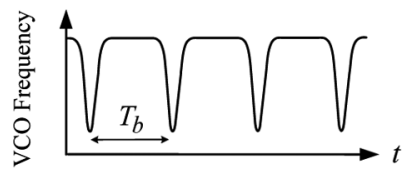

(b)

Fig. 3. Injection-pulling behavior of a VCO. (a) Fast-beat. (b) Quasi-lock.

\section{Frequency Pulling}

When injection signal beyond the locking range is impressed on the oscillator, i.e., $\left|\Delta \omega_{\text {osc }}\right|$ is larger than $\omega_{\mathrm{LR}}$, the pull-in process may never be accomplished and $\omega_{\text {out }}(t)$ will be modulated with $d \alpha(t) / d t$, which has an average beat note $\omega_{b}$. In this situation, the oscillator is under the injection-pulling condition and the average beat frequency is given as [12]

$$
\omega_{b}=\sqrt{\Delta \omega_{\mathrm{osc}}^{2}-\omega_{\mathrm{LR}}^{2}}
$$

Fig. 3 illustrates the injection-pulling behavior of a VCO using a series of correlated graphs. The discussion of injection pulling can be divided into fast-beat and quasi-lock, the two different conditions [12], as depicted in Fig. 3(a) and (b), respectively. In either one, the top graph shows the power versus frequency relation for the inherent oscillation signal $S_{\text {osc }}(t)$ and the injection signal $S_{\mathrm{inj}}(t)$. In this graph, the broken line represents the injection-locking characteristic curve of the oscillator, and $\omega_{\mathrm{LR}}$ is the locking range with respect to an injection signal power $P_{\text {inj. }}$. The middle graph shows the spectrum of the oscillator pulled by the injection signal. The oscillator output spectral lines are equally separated with a frequency spacing equal to $\omega_{b}$ given by (15). The bottom graph shows the oscillator output frequency $\omega_{\text {out }}(t)$ that is time varying with a period of $T_{b}=2 \pi / \omega_{b}$ during the injection-pulling process.

Fig. 3(a) illustrates the fast-beat condition with $\omega_{\text {inj }}$ far beyond the locking range, i.e., $\left|\omega_{\text {inj }}-\omega_{\text {osc }}\right|>\omega_{\text {LR }}$, whereas Fig. 3(b) illustrates the quasi-lock condition with $\omega_{\text {inj }}$ approaching close to the locking range, i.e., $\left|\omega_{\text {inj }}-\omega_{\text {osc }}\right| \rightarrow \omega_{\text {LR }}$. In fast-beat condition, the oscillator output spectral lines are separated with $\omega_{b}$ and have approximately equal levels at $\omega_{\text {inj }}$ and $\omega_{\text {inj }}-2 \omega_{b}$, as depicted in the middle graph of Fig. 3(a). In the quasi-lock condition, the oscillator output spectrum has a significant magnitude appearing at $\omega_{\mathrm{inj}}$, as depicted in the middle graph of Fig. 3(b). Furthermore, the bottom graphs in Fig. 3(a) and (b) displays that the oscillator output frequency $\omega_{\text {out }}(t)$ varies with an average beat cycle equal to $T_{b}$, and $T_{b}$ in the quasi-lock condition is longer than that in the fast-beat condition. This time-varying characteristic of the oscillator output frequency is useful to sense the existence of the injection signal in the proposed RF sensing circuit. 


\section{Synchronization Condition}

Since an oscillator can synchronize with a low-level injection signal, one of the popular applications for an injection-locked oscillator is to serve as a high gain amplifier [9], [11], [15], [16] and a frequency demodulator [17]-[19]. Recently, an injection-locked oscillator is used as a transponder to produce an approximately phase coherent high-power response to an interrogating signal delivered by a reader for local positioning application [20], [21]. As a high gain amplifier, the injection-locked oscillator can amplify an injected angle-modulated signal such as phase-modulated (PM) or frequency-modulated (FM ) signals that fit into the conditions $E_{\text {inj }}(t)=E_{\text {inj }}$ and $d \theta_{i}(t) / d t \neq 0$. In this section, we are concerned with an approach based on (7) to study an oscillator synchronizing with a general angle-modulated signal. When the oscillator is tuned to the injection frequency for amplifier applications and then synchronized with the injected angle-modulated signal, $\Delta \omega_{\text {osc }}=0$ and $d \alpha(t) / d t$ is equal to $d \theta_{i}(t) / d t$. It follows from (7) that

$$
\frac{d \alpha(t)}{d t}-\frac{d \theta_{i}(t)}{d t}=-B(t) \sin \alpha(t)-2 \frac{d \theta_{i}(t)}{d t}=0
$$

Substituting (8) into (16) and rearranging obtains

$$
\frac{d \theta_{i}(t)}{d t}=-\frac{1}{2}\left[\frac{\omega_{\mathrm{osc}}}{2 Q} \frac{E_{\mathrm{inj}}}{E_{\mathrm{osc}}} \frac{\sin \alpha(t)}{1+\frac{E_{\mathrm{inj}}}{E_{\mathrm{osc}}} \cos \alpha(t)}\right]
$$

where $E_{\text {inj }}$ indicates the amplitude of the constant envelope modulated signal. Since in the steady state the bracket term on the right-hand side of (17) has a maximum value equal to the locking range expressed in (13), accordingly we have

$$
\left|\frac{d \theta_{i}(t)}{d t}\right|<\frac{\omega_{\mathrm{LR}}}{2}
$$

Equation (18) indicates that the instantaneous modulation frequency cannot exceed half of the locking range to conform to the synchronization condition. Equation (18) further leads us to a consideration of practical PM or FM signals. First, we assume that an injection is a PM signal of the form [22]

$$
S_{P M}(t)=\cos \left(\omega_{\text {inj }} t+\theta_{i}(t)\right)
$$

where

$$
\theta_{i}(t)=\beta \cos \left(\omega_{m} t\right)
$$

is the instantaneous phase variation, $\omega_{m}$ is the modulation rate, and $\beta$ is the modulation index of the PM signal with a center

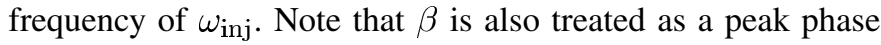
deviation $\Delta \phi_{\text {peak }}$ of the PM signal. Substituting (20) into (18) yields

$$
\left|\Delta \phi_{\text {peak }}\right|<\frac{1}{2} \frac{\omega_{\mathrm{LR}}}{\omega_{m}}
$$

Next, considering an injected FM signal by substituting modulation index $\beta$ in terms of $\Delta \omega_{\text {peak }} / \omega_{m}$ into (20) gives

$$
\left|\Delta \omega_{\text {peak }}\right|<\frac{\omega_{\mathrm{LR}}}{2}
$$

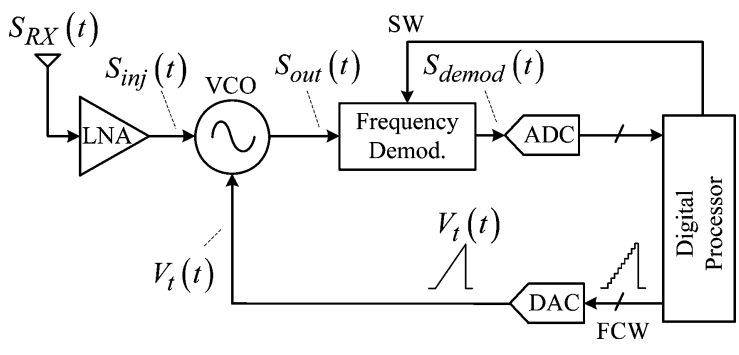

Fig. 4. Block diagram of the proposed RF sensing circuit.

where $\Delta \omega_{\text {peak }}$ represents the peak frequency deviation of the FM signal. This result coincides with the discussion in [9] that the distortion will be negligible if the peak frequency deviation of an FM signal is smaller than half of the locking range when the injection-locked oscillator is used as an FM amplifier. Finally, considering a digital angle-modulated signal with a modulation index $h$ and a bit rate $f_{b r}$, the signal has a peak frequency deviation given by [22]

$$
\Delta \omega_{\text {peak }}=\pi f_{b r} h
$$

Substituting (23) into (22) and rearranging obtains

$$
f_{b r}<\frac{1}{2 \pi} \frac{\omega_{\mathrm{LR}}}{h}
$$

The inequalities (21), (22), and (24) constrain the synchronization condition related to the locking range for PM, FM, and digital angle-modulated signals, respectively. The above derivations intend to provide the design criteria for the sensing circuit based on an injection-locked oscillator to support receiver detection of various constant-envelope modulated signals.

\section{SENSING ARCHITECTURE AND MECHANISM}

\section{A. System Architecture and Operation}

The block diagram of the proposed RF sensing circuit is shown in Fig. 4. The sensing circuit contains an antenna, a low-noise amplifier (LNA), a VCO with an additional injection input port, a frequency demodulator, and a digital processor with an analog-to-digital converter (ADC) and a digital-to-analog converter (DAC).

The RF sensing circuit can operate in two different modes governed by the digital processor via the switch (SW) control signal shown in Fig. 4. The first mode is to perform the spectrum scanning operation, whereas the other mode is to be a cognitive radio receiver. The sensing procedure is briefly demonstrated as follows. In the first place, the antenna receives a signal $S_{\mathrm{RX}}(t)$ from the environment and the LNA amplifies the received signal. The amplified signal $S_{\text {inj }}(t)$ is injected into a VCO via the injection input port. In the second place, the digital processor outputs a frequency control word (FCW) for the DAC to generate an analog sweeping voltage $V_{t}(t)$. The RF sensing circuit achieves its functionality through the use of $V_{t}(t)$ to tune the VCO inherent frequency and accomplish 


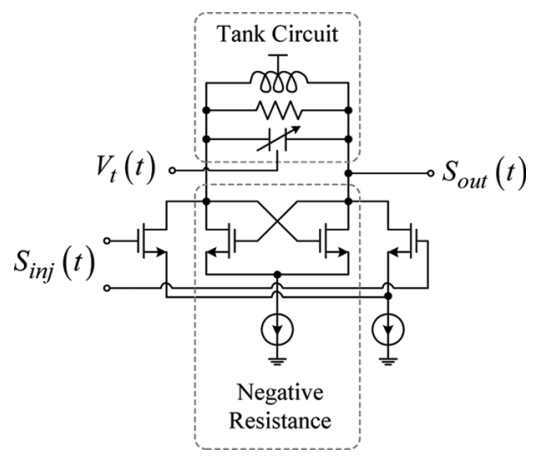

Fig. 5. Simplified circuit diagram of a VCO with an injection input.

the spectrum scanning operation in the aimed frequency range. Meanwhile, the frequency demodulator demodulates the VCO output signal $S_{\text {out }}(t)$ and the ADC samples the frequency-demodulated signal $S_{\text {demod }}(t)$ for the digital processor to detect the signal. The sensing procedure diagnoses the signal activities in the spectrum and records the corresponding FCWs. After the spectrum sensing procedure completes, the digital processor can switch the sensing circuit to serve as a receiver and establish communications in unoccupied frequencies for cognitive radios.

\section{B. Sensing Principle}

A simplified circuit diagram of the VCO used in the sensing circuit is shown in Fig. 5. The VCO is composed of a tank circuit controlled by a tuning voltage $V_{t}(t)$ and a differential amplifier to generate a negative resistance for oscillation. An additional differential pair of transistors is used to receive the injection signal $S_{\text {inj }}(t)$. $S_{\text {out }}(t)$ is the VCO output signal under tuning and injection condition.

In Section II, we examined the oscillator behavior under injection only at a single free-running frequency. Several observations have shown that the oscillator output frequency $\omega_{\text {out }}(t)$ is modulated with an average beat note $\omega_{b}$ under the injection-pulling condition and the value of $\omega_{b}$ can be estimated according to (15). However, in RF sensing applications, the oscillator inherent frequency is voltage controlled and $\omega_{\text {osc }}$ is tuned to sweep across the aimed sensing frequency range by applying a sweeping voltage $V_{t}(t)$. When the sensing circuit performs the scanning operation, a modulation signal can be regarded as a relatively narrowband signal under a fast VCO inherent frequency sweeping speed, i.e., $100 \mathrm{MHz} / \mathrm{ms}$ in our case. Therefore, it is reasonable to assume that the modulation envelope and phase remain almost time invariant, i.e., $E_{\text {inj }}(t) \approx E_{\text {inj }}$ and $d \theta_{i}(t) / d t \approx 0$, when the oscillator inherent frequency sweeps across the signal bandwidth with such a fast speed.

Taking an instantaneous tuning voltage $V_{t}(t)$ into account, (9) can be rewritten as

$$
\Delta \omega_{\mathrm{osc}}(t)=\omega_{\mathrm{osc}}(t)-\omega_{\mathrm{inj}}=\omega_{c}+K_{V} V_{t}(t)-\omega_{\mathrm{inj}}
$$

where $K_{V}$ is the tuning sensitivity and $\omega_{c}$ is the center frequency of the VCO. As the VCO inherent frequency is sweeping with

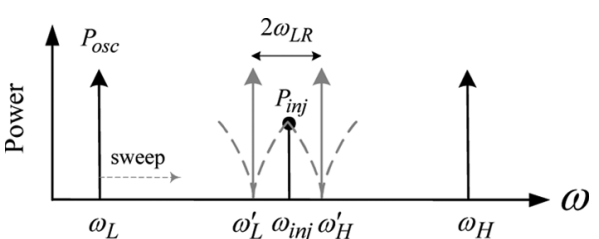

(a)

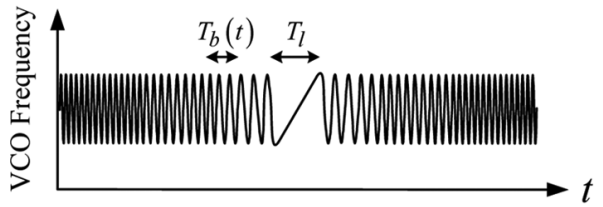

(b)

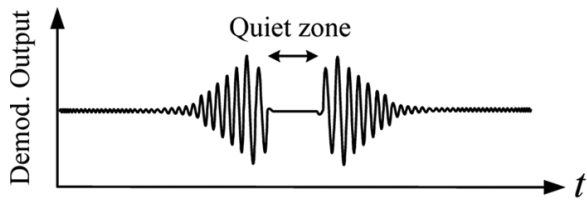

(c)

Fig. 6. Illustrations of the sensing principle. (a) Power versus frequency relation for the inherent oscillation signal and the injection signal. (b) VCO frequency pulled by injection under frequency sweeping. (c) Bandpass filtering of the frequency-demodulated output.

time, the average beat frequency can be obtained by replacing $\Delta \omega_{\text {osc }}$ in (13) and (15) with $\Delta \omega_{\text {osc }}(t)$ and shown as

$$
\omega_{b}(t)=\sqrt{\Delta \omega_{\mathrm{osc}}^{2}(t)-\left[\frac{\omega_{\mathrm{inj}}+\Delta \omega_{\mathrm{osc}}(t)}{\left.2 Q \sqrt{1-\left(\frac{E_{\mathrm{inj}}}{E_{\mathrm{osc}}}\right)^{2}} \frac{E_{\mathrm{inj}}}{E_{\mathrm{osc}}}\right]^{2}} .\right.}
$$

It is clear from (26) that $\omega_{b}(t)$ and $T_{b}(t)$ vary with $\Delta \omega_{\text {osc }}(t)$ under the injection-pulling condition when the VCO inherent frequency sweeps.

Fig. 6 explains the RF sensing principle using injection locking and frequency demodulation. In Fig. 6(a), $\omega_{\text {osc }}(t)$ is assumed to continuously sweep from a lowest frequency $\omega_{L}$ to a highest frequency $\omega_{H}$ of the sensing frequency range. $\omega_{L}^{\prime}$ and $\omega_{H}^{\prime}$ is the lower and higher VCO inherent frequency, respectively, with a frequency offset from the injection frequency $\omega_{\text {inj }}$ by a locking range $\omega_{\mathrm{LR}}$, i.e., $\left(\omega_{H}^{\prime}-\omega_{L}^{\prime}\right)=2 \omega_{\mathrm{LR}}$.

As Fig. 6(b) illustrates, at the beginning, in the period of tuning $\omega_{\text {osc }}(t)$ from $\omega_{L}$ to $\omega_{L}^{\prime}$, the VCO output frequency is FM with a time-varying beat cycle $T_{b}(t)$ since the VCO experiences the two different injection-pulling conditions, first the fast beat and then the quasi-lock. Next, in the period of tuning $\omega_{\text {osc }}(t)$ from $\omega_{L}^{\prime}$ to $\omega_{H}^{\prime}, \omega_{\text {inj }}$ falls in the locking range. From (2), the instantaneous beat frequency $d \alpha(t) / d t$ is getting smaller as the VCO output frequency approaches the injection frequency more closely. Substituting (7) and (25) into (2) with $E_{\text {inj }}(t) \approx E_{\text {inj }}$ and $d \theta_{i}(t) / d t \approx 0$, we can express the instantaneous VCO output frequency as

$$
\omega_{\text {out }}(t) \approx\left[\omega_{c}+K_{V} V_{t}(t)\right] \cdot\left[1-\frac{\sin \alpha(t)}{2 Q\left(\frac{E_{\text {osc }}}{E_{\text {inj }}}+\cos \alpha(t)\right)}\right] .
$$




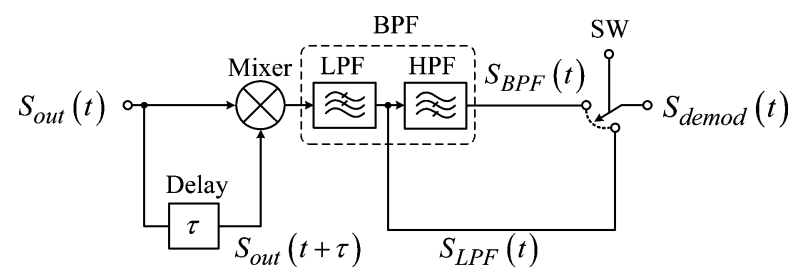

Fig. 7. Block diagram of the frequency demodulator.

Since $d \alpha(t) / d t$ is a smaller value in the locking range, the phase $\alpha(t)$ is close to a constant, and hence, the second bracket of the product on the right-hand side of (27) can be treated as an approximate constant. Therefore, in this tuning period, the instantaneous VCO output frequency $\omega_{\text {out }}(t)$ varies linearly with the instantaneous tuning voltage $V_{t}(t)$. As a result, a linear frequency versus time region with a time period of $T_{l}$ is obtained, as shown in Fig. 6(b), when a linear sweeping voltage is applied as the tuning voltage. At last, in the period of tuning $\omega_{\text {osc }}(t)$ from $\omega_{H}^{\prime}$ to $\omega_{H}$, the VCO output frequency is again FM with a time-varying beat cycle $T_{b}(t)$ due to experience with the two different injection-pulling conditions in a reverse order.

Fig. 6(c) shows the results when the VCO output signal is frequency demodulated and subsequently bandpass filtered. The aim of this signal processing is to produce a narrowband signal containing sufficient information for determining the frequency and power of the sensed signal. This can be further accomplished by detecting the quiet zone in the demodulated output waveform, as shown in Fig. 6(c), with the use of a low sampling rate $\mathrm{ADC}$ and a low data rate digital processor.

\section{Frequency Demodulation}

Fig. 7 shows the block diagram of the designed frequency demodulator in the sensing circuit. The demodulator contains a delay element, a mixer, a bandpass filter composed of a low-pass filter (LPF) and a high-pass filter (HPF), and a switch controlled by the digital processor via the SW signal. The VCO output signal $S_{\text {out }}(t)$ can be represented as

$$
\begin{aligned}
S_{\text {out }}(t) & =E_{\text {out }} \cos \left(\omega_{\text {out }}(t) t+\theta_{0}\right) \\
& =E_{\text {out }} \cos \left(\omega_{c} t+\phi_{m}(t)\right)
\end{aligned}
$$

where $E_{\text {out }}$ is the output amplitude, $\omega_{\text {out }}(t)$ is the instantaneous output frequency, $\theta_{0}$ is the initial phase of the VCO, $\omega_{c}$ is the center frequency of the $\mathrm{VCO}$, and $\phi_{m}(t)$ is defined as the oscillator phase modulation function. With (2), (7), and (25), $\phi_{m}(t)$ in (28) can be formulated as

$$
\phi_{m}(t)=\phi_{s}(t)+\phi_{\alpha}(t)
$$

where

$$
\phi_{s}(t)=K_{V} \int_{t_{0}}^{t} V_{t}\left(t^{\prime}\right) d t^{\prime}+\theta_{0}
$$

expresses the VCO phase variation caused by VCO inherent frequency sweeping, and

$$
\phi_{\alpha}(t)=-\int_{t_{0}}^{t} B\left(t^{\prime}\right) \sin \alpha\left(t^{\prime}\right) d t^{\prime}
$$

represents the phase modulation caused by the injection signal under frequency sweeping. As shown in Fig. 7, the mixer multiplies $S_{\text {out }}(t)$ by its delay version $S_{\text {out }}(t+\tau)$ and outputs to the LPF to reject high-order intermodulation components. The low-pass filtering signal $S_{\mathrm{LPF}}(t)$ is approximately given by

$$
\begin{aligned}
S_{\mathrm{LPF}}(t) \approx \frac{E_{\mathrm{LPF}}^{2}}{2} \cos \left(\omega_{c} \tau\right. & +K_{V} \int_{t}^{t+\tau} V_{t}\left(t^{\prime}\right) d t^{\prime} \\
& \left.+\theta_{0}-\int_{t}^{t+\tau} B\left(t^{\prime}\right) \sin \alpha\left(t^{\prime}\right) d t^{\prime}\right) .
\end{aligned}
$$

Assuming that the delay element $\tau$ is reasonably small obtains

$$
\begin{aligned}
S_{\mathrm{LPF}}(t) \approx \frac{E_{\mathrm{LPF}}^{2}}{2} \cos \left(\omega_{c} \tau+K_{V}\right. & V_{t}(t) \tau-B(t) \\
\times & \left.\times \sin \alpha(t) \tau+\theta_{0}\right) .
\end{aligned}
$$

The LPF can help the sensing circuit to reject high-frequency beat notes to relax the ADC requirements. The low-pass filtered $S_{\mathrm{LPF}}(t)$ further passes through the following HPF to eliminate the low frequency-tuning component $K_{V} V_{t}(t) \tau$ in (33) for producing a waveform depicted in Fig. 6(c), which is mainly determined by the bandpass filtered beat notes caused by the injection signal.

When the sensing circuit performs spectrum scanning operation, the digital processor switches $S_{\mathrm{BPF}}(t)$ to $S_{\text {demod }}(t)$ for the ADC to sample the demodulated output, which has a quiet zone between two oscillating wavelets, as shown in Fig. 6(c). When the sensing circuit operates as a receiver, the tuning voltage $V_{t}(t)$ tunes the $\mathrm{VCO}$ to a recognized cognitive radio signal frequency and then remains a constant voltage. The digital processor switches $S_{\mathrm{LPF}}(t)$ to $S_{\text {demod }}(t)$ for ADC to sample the demodulated output, which contained the received modulation information. The HPF is bypassed to preserve low-frequency components of the modulation information.

\section{Frequency and Power Detection}

Consider that $\omega_{\text {osc }}(t)$ is sweeping over an aimed bandwidth $\omega_{\mathrm{bw}}=\left(\omega_{H}-\omega_{L}\right)$ in a time period $T_{\mathrm{swp}}$, the frequency sweeping rate $\mathrm{SR}$ can then be defined as

$$
\mathrm{SR}=\frac{1}{2 \pi} \frac{\omega_{\mathrm{bw}}}{T_{\mathrm{swp}}}=K_{V} \frac{V_{\mathrm{swp}}}{T_{\mathrm{swp}}}\left(\frac{\mathrm{Hz}}{\mathrm{s}}\right)
$$

where $V_{\text {swp }}$ is the tuning voltage amplitude for the VCO frequency to cover the bandwidth. The time period $T_{l}$ of the quiet zone, as depicted in Fig. 6(c), is equal to

$$
T_{l}=\frac{\left(\omega_{H}^{\prime}-\omega_{L}^{\prime}\right)}{2 \pi \cdot \mathrm{SR}}=\frac{\omega_{\mathrm{LR}}}{\pi \cdot \mathrm{SR}} .
$$

After the VCO output frequency is demodulated, the digital processor can detect the signal activity by using the quiet-zone characteristic. Furthermore, the digital processor can record the 
FCW corresponding to the middle of the quiet zone in the recognition process. According to (13) and (35), the injection level $E_{\text {inj }}$ inputting to the VCO can be obtained as

$$
E_{\text {inj }}=\frac{E_{\text {osc }}}{\sqrt{\left(\frac{\omega_{\text {osc }}}{2 \pi \cdot \mathrm{SR} \cdot Q \cdot T_{l}}\right)^{2}+1}} .
$$

The above equation indicates that the injection level can be calculated from the measured $T_{l}$. The actual signal level $S_{\mathrm{RX}}(t)$ at the sensing circuit input can be further obtained from knowledge of the LNA power gain.

\section{Discrete Time ANalysis}

To predict the behavior of the RF sensing circuit facing a practical modulation signal, a discrete-time dynamic model was established based on (7) derived in Section II. To begin with, we first substitute a time-varying $\Delta \omega_{\text {osc }}(t)$ into (7) and make a discretization with respect to time to obtain

$$
\begin{array}{r}
\frac{\alpha\left(t_{i}\right)-\alpha\left(t_{i-1}\right)}{\Delta t}=-B\left(t_{i}\right) \sin \alpha\left(t_{i}\right)+\Delta \omega_{\mathrm{osc}}\left(t_{i}\right) \\
-\frac{\theta\left(t_{i}\right)-\theta\left(t_{i-1}\right)}{\Delta t}
\end{array}
$$

where $\Delta t=\left(t_{i}-t_{i-1}\right)$ is the calculation time period, and

$$
\begin{aligned}
B\left(t_{i}\right) & =\frac{\omega_{\mathrm{osc}}\left(t_{i}\right)}{2 Q} \frac{E_{\mathrm{inj}}\left(t_{i}\right)}{E_{\mathrm{osc}}} \frac{1}{1+\frac{E_{\mathrm{inj}}\left(t_{i}\right)}{E_{\mathrm{osc}}} \cos \alpha\left(t_{i}\right)} \\
\Delta \omega_{\mathrm{osc}}\left(t_{i}\right) & =\omega_{\mathrm{osc}}\left(t_{i}\right)-\omega_{\mathrm{inj}}=\omega_{c}+K_{V} V_{t}\left(t_{i}\right)-\omega_{\mathrm{inj}} .
\end{aligned}
$$

When $\Delta t$ is small, $\alpha\left(t_{i}\right)$ is approximately equal to $\alpha\left(t_{i-1}\right)$. Replacing $\alpha\left(t_{i}\right)$ by $\alpha\left(t_{i-1}\right)$ in the right-hand side of (37) and (38) and rearranging gives

$$
\begin{aligned}
\alpha\left(t_{i}\right)= & {\left[-B\left(t_{i}\right) \sin \alpha\left(t_{i-1}\right)+\Delta \omega_{\mathrm{osc}}\left(t_{i}\right)\right] \Delta t } \\
& -\theta\left(t_{i}\right)+\theta\left(t_{i-1}\right)+\alpha\left(t_{i-1}\right) \\
B\left(t_{i}\right)= & \frac{\omega_{\mathrm{osc}}\left(t_{i}\right)}{2 Q} \frac{E_{\mathrm{inj}}\left(t_{i}\right)}{E_{\mathrm{osc}}} \frac{1}{1+\frac{E_{\mathrm{inj}}\left(t_{i}\right)}{E_{\mathrm{osc}}} \cos \alpha\left(t_{i-1}\right)} .
\end{aligned}
$$

According to (40) and (41), $\alpha\left(t_{i}\right)$ can recursively be calculated instead of solving (37) at time instant $t_{i}$. We further rewrite (40) with an index $n$ to represents $\alpha\left(t_{i}\right)$ as a combination of data sequences in the discrete-time domain [23]

$$
\begin{aligned}
\alpha[n]= & \left(-B[n] \cdot \sin \alpha[n-1]+\Delta \omega_{\mathrm{osc}}[n]\right) T \\
& -\theta[n]+\theta[n-1]+\alpha[n-1] \\
B[n]= & \frac{\omega_{\mathrm{osc}}[n]}{2 Q} \frac{E_{\mathrm{inj}}[n]}{E_{\mathrm{osc}}} \frac{1}{1+\frac{E_{\mathrm{inj}}[n]}{E_{\mathrm{osc}}} \cos \alpha[n-1]} \\
\Delta \omega_{\mathrm{osc}}[n]= & \omega_{\mathrm{osc}}[n]-\omega_{\mathrm{inj}}=\omega_{c}+K_{V} V_{t}[n]-\omega_{\mathrm{inj}}
\end{aligned}
$$

where $T=\Delta t$ represents the calculation time-step.

Referring to (28), the VCO output can be viewed as a signal PM by a function $\phi_{m}(t)$ shown in (29). In the discrete-time domain, this oscillator phase modulation function can be represented as

$$
\phi_{m}[n]=K_{v} V_{t}[n] \cdot n T+\theta_{0}+\phi_{\alpha}[n]
$$

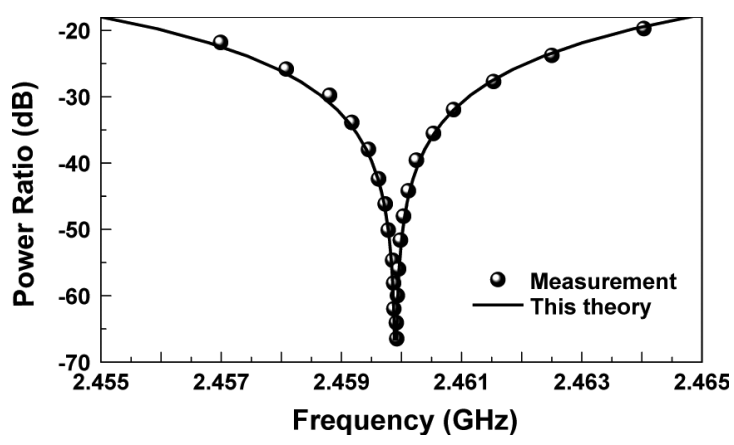

Fig. 8. Comparison of the VCO injection-locking characteristics between theory and measurement.

where

$$
\phi_{\alpha}[n]=\phi_{\alpha}[n-1]-B[n] \cdot \sin \alpha[n] \cdot T .
$$

The phase modulation function is finally expressed as a pulse train weighted by $\phi_{m}[n]$ expressed in (45). The VCO output waveform can be reconstructed using the form [23]

$$
S_{\text {out }}(t)=E_{\text {out }} \cos \left(\omega_{c} t+\sum_{n} \phi_{m}[n] \cdot h_{r}(t-n T)\right)
$$

where $h_{r}(t)$ is the impulse response of the reconstruction filter. The VCO output spectrum can then be predicted by taking the Fourier transform of (47).

\section{COMPUTED AND EXPERIMENTAL RESUlTS}

This section shows the computed and experimental results of the presented RF sensing circuit. The VCO is designed with an injection input port and has an output power of $1.44 \mathrm{dBm}$ at $2.46 \mathrm{GHz}$. The tuning range of the VCO covers from 2.4 to $2.484 \mathrm{GHz}$ with a tuning voltage $V_{t}$ ranging from 0 to $2.8 \mathrm{~V}$. The tuning sensitivity $K_{V}$ is $30 \mathrm{MHz} / \mathrm{V}$. Fig. 8 shows the theoretical and measured characteristics of VCO injection locking at a free-running oscillation frequency of $2.46 \mathrm{GHz}$. Note that the theoretical results are tuned to best fit the measured ones by estimating the tank $Q$ factor as 32.8. The VCO in the presented RF sensing circuit can detect a minimum power of $-67 \mathrm{dBm}$ using the injection-locking technique.

The following sections demonstrate the computed and experimental results for the RF sensing circuit to perform spectrum sensing and to serve as a cognitive radio receiver. In the spectrum sensing experiment, $V_{t}(t)$ was linearly sweeping from 0 to $2.8 \mathrm{~V}$ in $0.84 \mathrm{~ms}$ to tune the $\mathrm{VCO}$ frequency from 2.4 to $2.484 \mathrm{GHz}$, leading to a sweeping rate equal to $100 \mathrm{MHz} / \mathrm{ms}$. The first sensing experiment was set for the RF sensing circuit to sense a sinusoidal signal at $2.46 \mathrm{GHz}$ with various injection power. The second sensing experiment was set for the RF sensing circuit to sense a combination of three independent modulation signals. In the receiver experiment, the VCO frequency is tuned to a constant value allocated by the digital processor, and the sensing circuit was set to serve as a cognitive radio receiver. 

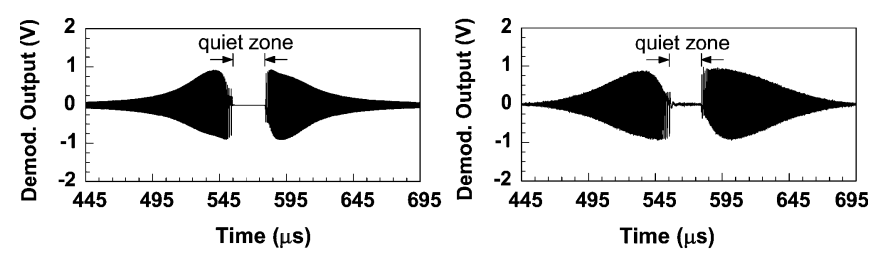

(a)
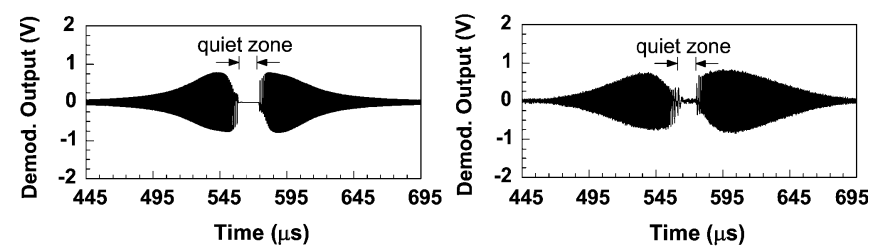

(b)
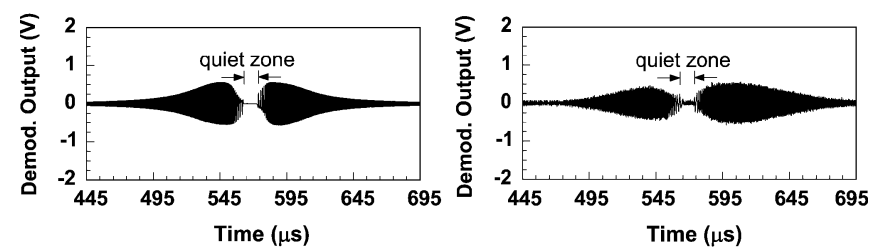

(c)

Fig. 9. Comparison of demodulated output waveform of the RF sensing circuit between computation and measurement in the sensing of a sinusoidal signal with various injection power. (a) $P_{\mathrm{inj}}=-28.5 \mathrm{dBm}$. (b) $P_{\mathrm{inj}}=-33.56 \mathrm{dBm}$. (c) $P_{\text {inj }}=-38.56 \mathrm{dBm}$. (a) (left) computed, (right) measured. (b) (left) computed, (right) measured. (c) (left) computed, (right) measured.

TABLE I

COMPARISONS OF DETECTED QUIET-Zone DURATIONS

\begin{tabular}{c|c|c|c}
\hline \hline Injection Power & $-28.56 \mathrm{dBm}$ & $-33.56 \mathrm{dBm}$ & $-38.56 \mathrm{dBm}$ \\
\hline Measured & $22.4 \mu \mathrm{s}$ & $12.3 \mu \mathrm{s}$ & $7.4 \mu \mathrm{s}$ \\
\hline Estimated & $22.6 \mu \mathrm{s}$ & $12.7 \mu \mathrm{s}$ & $7.14 \mu \mathrm{s}$ \\
\hline \hline
\end{tabular}

\section{A. Sinusoidal Signal Sensing Results}

To verify the proposed discrete-time dynamic model for the sensing behavior of the VCO, the experiment bypassed the LNA to directly inject signals into the VCO in the RF sensing circuit. The experiment was set for the VCO to sense a sinusoidal signal at $2.46 \mathrm{GHz}$ with various injection signal power. Fig. 9 compares the computed and measured results of the demodulated output waveform. Fig. 9(a)-(c) shows the demodulated output results under injection signal power of $-28.56,-33.56$, and $-38.56 \mathrm{dBm}$, respectively. The computed and measured results both show a quiet zone with different durations under different injection power; the duration goes narrower when the injection power decreases. The estimated and experimental results of the quiet-zone duration are summarized in Table I. These results are in good agreement, both showing a quite-zone duration of about 22,12 , and $7 \mu$ s, respectively.

\section{B. Modulation Signal Sensing Results}

This section demonstrates the results for the RF sensing circuit to sense a combination of three independent modulation signals. The spectrum of the combined signal is shown in Fig. 10. The first one is an FM signal located at $f_{1}=2.427 \mathrm{GHz}$ with a power $P_{1}=-80 \mathrm{dBm}$ and $320-\mathrm{kHz}$ modulation bandwidth; the second one is a Gaussian minimum shift-keying (GMSK) signal

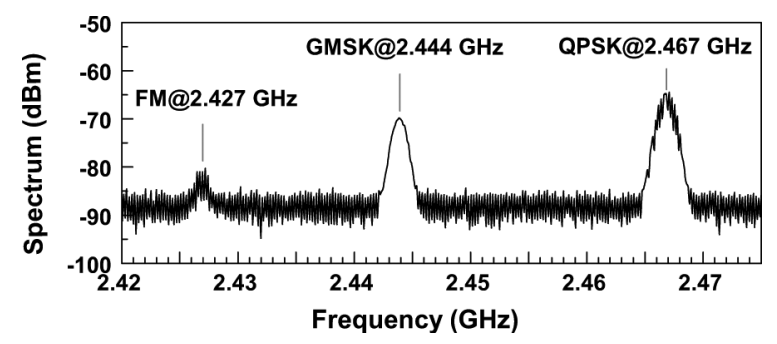

Fig. 10. Spectrum of three signals including a $2.427-\mathrm{GHz}$ FM signal, a 2.444-GHz GMSK signal, and a 2.467-GHz QPSK signal.

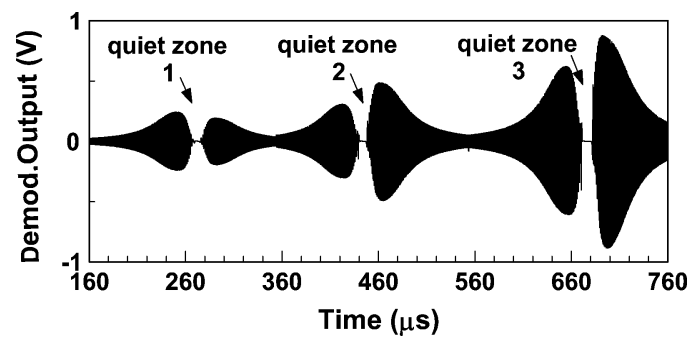

Fig. 11. Computed demodulated output waveform of the RF sensing circuit in the sensing of the spectrum shown in Fig. 10.

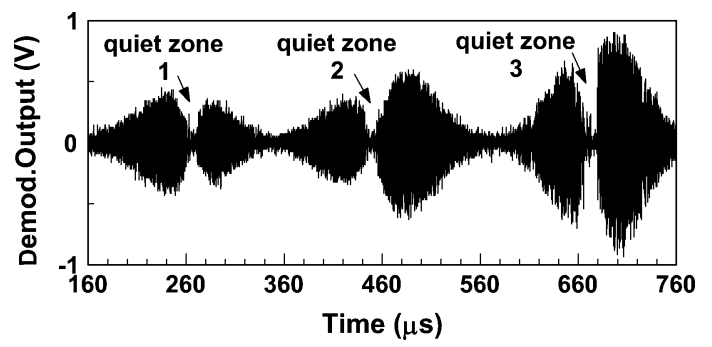

Fig. 12. Measured demodulated output waveform of the RF sensing circuit in the sensing of the spectrum shown in Fig. 10.

TABLE II

COMPARISONS OF ESTIMATED SPECTRUM SENSING RESUlTS

\begin{tabular}{l|c|c|c}
\hline \hline & $\begin{array}{c}\text { Measured results } \\
\text { in Fig. 10 }\end{array}$ & $\begin{array}{c}\text { Estimated results } \\
\text { from Fig. 11 }\end{array}$ & $\begin{array}{c}\text { Estimated results } \\
\text { from Fig. 12 }\end{array}$ \\
\hline$f_{1}(\mathrm{GHz})$ & 2.427 & 2.427 & 2.4262 \\
\hline$P_{1}(\mathrm{dBm})$ & -80 & -72.4 & -71.9 \\
\hline \hline$f_{2}(\mathrm{GHz})$ & 2.444 & 2.444 & 2.4446 \\
\hline$P_{2}(\mathrm{dBm})$ & -70 & -67.1 & -66.39 \\
\hline \hline$f_{3}(\mathrm{GHz})$ & 2.467 & 2.467 & 2.4668 \\
\hline$P_{3}(\mathrm{dBm})$ & -65 & -64.1 & -62.87 \\
\hline \hline
\end{tabular}

located at $f_{2}=-2.444 \mathrm{GHz}$ with a power $P_{2}=-70 \mathrm{dBm}$ and $271 \mathrm{~kb} / \mathrm{s}$ data rate; the third one is a quadrature phase-shift keying (QPSK) signal located at $f_{3}=2.467 \mathrm{GHz}$ with a power $P_{3}=-65 \mathrm{dBm}$ and $1-\mathrm{Mb} / \mathrm{s}$ data rate. The combined signal was first amplified by an LNA with $28-\mathrm{dB}$ power gain and then injected to the VCO. Figs. 11 and 12 show, respectively, the computed and experimental results of the RF sensing circuit demodulation output. The detected frequency and power are individually estimated according to the computed and experimental results, and these estimations are summarized in Table II. The estimated injection frequencies from computation and experiment 


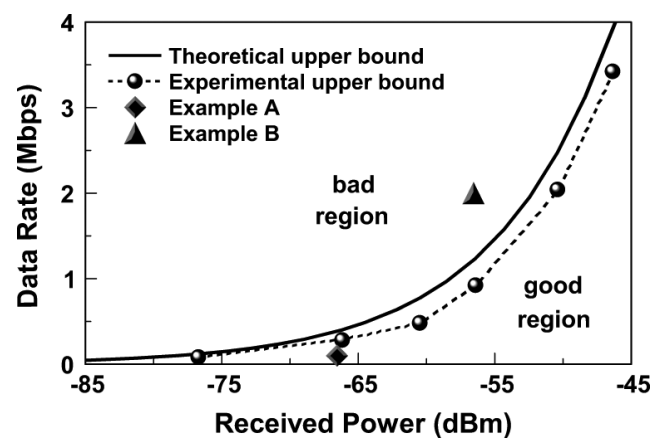

Fig. 13. Applicable range of data rate versus received power for the RF sensing circuit to perform the receiver detection.

are close to the measured ones with an error less than $1 \mathrm{MHz}$. Due to the influence of noise and demodulator performance, the estimated results are a little bit less accurate in power, especially with a low power injection. However, it can be seen that the presented circuit is capable of simultaneously sensing multiple signals by detecting the individual quiet-zone durations. This capability makes the proposed RF sensing circuit more attractive in practical cognitive radio applications.

\section{Receiver Detection Results}

After the spectrum scanning operation is accomplished, the $\mathrm{RF}$ sensing circuit tunes the VCO to a specific frequency to serve as a receiver. In this experiment, a GMSK signal is assumed as a received signal for cognitive radio applications. The communication can support various data rates and power levels, and the maximum rating is constrained by (24) for a GMSK signal with a modulation index $h$ equal to 0.5 . Fig. 13 shows the applicable range of data rate versus received power for the RF sensing circuit to perform the receiver detection. The solid line is the theoretical result from (24) that bounds the maximum usable data rate. The dotted line is the measured maximum data rate with the criterion of no significant degradation (i.e., within the equipment error range). It can be seen that the maximum data rate of a received signal increases with the received power. Note that this will not be a problem in the application because the data rate can be dynamically adjusted according to the received power for cognitive communications. Fig. 13 shows that the experimental upper bounds of data rate slightly deviate from the theoretical ones. This is because the VCO inherent frequency is adrift in practice while measuring. In addition, the time constant for an oscillator to reach the steady state are not considered in theoretical prediction.

Two experimental examples for receiver detection are also shown in Fig. 13. Example A is a GMSK signal with $-66.56 \mathrm{dBm}$ power and $100-\mathrm{kb} / \mathrm{s}$ data rate, which is within the upper bound of data rate. In contrast, example B is with $-56.56 \mathrm{dBm}$ power and $2-\mathrm{Mb} / \mathrm{s}$ data rate, which is beyond the upper bound of data rate. The demodulated signal constellation and eye diagrams of example A and B are shown in Fig. 14(a) and (b), respectively. The measured error vector magnitude (EVM) for example A is $1.39 \%$, while the result for example $\mathrm{B}$ is $22.59 \%$. These two examples evidence that the sensing circuit can perform the receiver detection well only within the good region shown in Fig. 13.

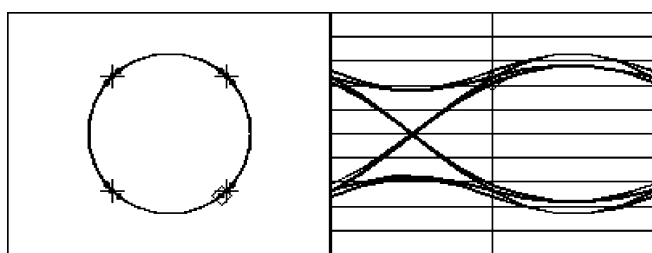

(a)

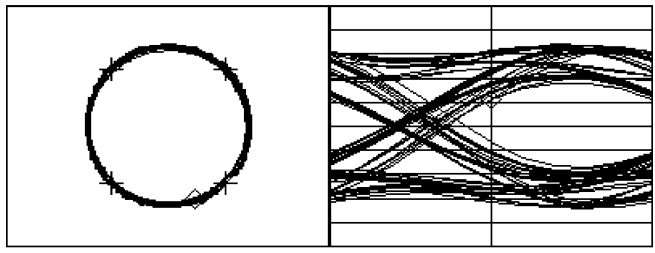

(b)

Fig. 14. Demodulated signal constellation and eye diagrams of the two receiver detection examples shown in Fig. 13. (a) Example A with a measured EVM of $1.39 \%$. (b) Example B with a measured EVM of $22.59 \%$. (a) (left) constellation diagram, (right) eye diagram. (b) (left) constellation diagram, (right) eye diagram.

\section{CONCLUSION}

This paper has presented a prototype of RF sensing circuit that is capable of sensing sinusoidal signals and modulation signals for cognitive radio applications. This paper has also provided the detailed theory and numerical computations for the circuit to explain the sensing mechanism, as well as predict the sensing performance in a comprehensive manner. The experiment shows that the circuit can sense a signal power as low as $-80 \mathrm{dBm}$. This sensitivity can be further improved with a higher gain LNA and a variable power VCO. The proposed RF sensing circuit has a spectrum scanning speed equal to $100 \mathrm{MHz} / \mathrm{ms}$, showing a more attractive performance versus complexity benefit than the usual approaches using a frequency synthesizer. In addition, the same circuit can also serve as a cognitive radio receiver supporting variable data rate under received-power constraints.

\section{ACKNOWLEDGMENT}

The authors wish to thank the National Chip Implementation Center, Taiwan, for providing the CMOS foundry service for this study.

\section{REFERENCES}

[1] "Spectrum policy task force report," FCC, Washington, DC, ET Docket No. 02-115, 2002.

[2] "Unlicensed operation in the TV broadcast bands and additional spectrum for unlicensed devices below $900 \mathrm{MHz}$ in the $3 \mathrm{GHz}$ band," FCC, Washington, DC, 2004, Notice of proposed rulemaking FCC 04-113.

[3] J. Mitola, III and G. Q. Maguire, Jr, "Cognitive radio: Making software radios more personal," IEEE Per. Commun., vol. 6, no. 4, pp. 13-18, Aug. 1999.

[4] S. M. Mishra, D. Cabric, C. Chang, D. Willkomm, B. Schewick, A. Wolisz, and R. W. Brodersen, "A real time cognitive radio testbed for physical and link layer experiments," in Proc. 1st IEEE Int. New Frontier in Dynamic Spectrum Access Networks Symp., Baltimore, MD, 2005 , pp. 562-567.

[5] A. Mayer et al., "RF front-end architecture for cognitive radios," in Proc. 18th Annu. Personal, Indoor. Mobile Radio Commun. Symp., Athens, Greece, 2007, pp. 1-5.

[6] B. Ackland and I. Seskar, "High performance cognitive radio platform with integrated physical \& network layer capabilities," presented at the Berkeley Wireless Res. Center Cognitive Radio Workshop, Berkeley, CA, Nov. 1, 2004 
[7] C.-J. Li, F. K. Wang, T.-S. Horng, and K. C. Peng, "A novel RF sensing circuit using injection locking and frequency demodulation for cognitive radio applications," in IEEE MTT-S Int. Microw. Symp. Dig., 2009, pp. 1165-1168.

[8] R. Adler, "A study of locking phenomena in oscillators," Proc. IRE, vol. 34, no. 6, pp. 351-357, Jun. 1946.

[9] R. D. Huntoon and A. Weiss, "Synchronization of oscillators," Proc. IRE, vol. 35 , no. 12 , pp. 1415-1423, Dec. 1947.

[10] L. J. Paciorek, "Injection locking of oscillators," Proc. IEEE, vol. 53, no. 11 , pp. 1723-1727, Nov. 1965

[11] K. Kurokawa, "Injection locking of microwave solid-state oscillators," Proc. IEEE, vol. 61, no. 10, pp. 1386-1410, Oct. 1973.

[12] B. Razavi, "A study of injection locking and pulling in oscillators," IEEE J. Solid-State Circuits, vol. 39, no. 9, pp. 1415-1424, Sep. 2004.

[13] A. Mirzaei, M. E. Heidari, and A. A. Abidi, "Analysis of oscillators locked by large injection signals: Generalized Adler's equation and geometrical interpretation," in Proc. IEEE Custom Integr. Circuits Conf., San Jose, CA, 2006, pp. 737-740.

[14] G. D. Vendelin, A. M. Pavio, and U. L. Rohde, Microwave Circuit Design Using Linear and Nonlinear Techniques. New York: Wiley, 1990, ch. 6

[15] R. C. Mackey, "Injection locking of klystron oscillators," IRE Trans. Microw. Theory Tech., vol. MTT-10, no. 4, pp. 228-235, Jul. 1962.

[16] H. L. Stover and R. C. Shaw, "Injection-locked oscillators as amplifiers for angle-modulated signals," in IEEE MTT-S Int. Microw. Symp. Dig., 1966, pp. 60-66.

[17] E. Main and D. Coffing, "FM demodulation using an injection-locked oscillator," in IEEE MTT-S Int. Microw. Symp. Dig., 2000, pp. $135-138$.

[18] E. Main and D. Coffing, "An FSK demodulator for bluetooth applications having no external components," IEEE Trans. Circuits Syst. II, Analog Digit. Process., vol. 49, no. 6, pp. 373-378, Jun. 2002.

[19] F. Ramírez, V. A. Araña, and A. Suárez, "Frequency demodulator using an injection-locked oscillator: Analysis and design," IEEE Microw. Wireless Compon. Lett., vol. 18, no. 1, pp. 43-45, Jan. 2008.

[20] M. Vossiek and P. Gulden, "The switched injection-locked oscillator: A novel versatile concept for wireless transponder and localization systems," IEEE Trans. Microw. Theory Tech., vol. 56, no. 4, pp. 859-866, Apr. 2008.

[21] S. Wehrli, D. Barras, F. Ellinger, and H. Jäckel, "Integrated active pulsed reflector for FMCW radar localization," in IEEE MTT-S Int. Microw. Symp. Dig., 2009, pp. 81-84.

[22] S. Haykin, Communication Systems, 4th ed. New York: Wiley, 2001.

[23] A. V. Oppenheim, R. W. Schafer, and J. R. Buck, Discrete-Time Signal Processing, 2nd ed. Upper Saddle River, NJ: Prentice-Hall, 1999.

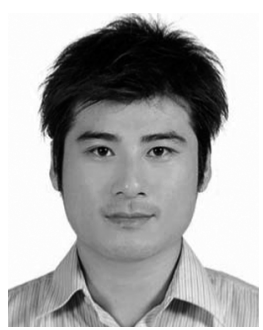

Chien-Jung Li (S'07) was born in Tainan, Taiwan, on October 26, 1979. He received the B.S.E.E. and $\mathrm{Ph} . \mathrm{D}$. degrees from National Sun Yat-sen University, Kaohsiung, Taiwan, in 2002 and 2009, respectively.

$\mathrm{He}$ is currently a Post-Doctoral Researcher with the Department of Electrical Engineering, National Sun Yat-sen University. His research interests include PA linearization techniques, frequency synthesizer designs, and local oscillator pulling issues in direct-conversion transceivers.

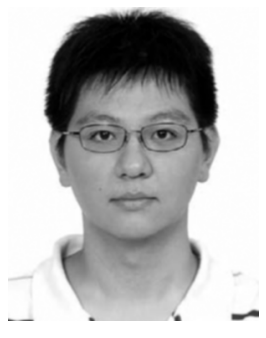

Fu-Kang Wang was born in Kaohsiung, Taiwan, on May 15, 1985. He received the B.S.E.E. and M.S.E.E. degrees from National Sun Yat-sen University, Kaohsiung, Taiwan in 2007 and 2009, respectively, and is currently working toward the Ph.D. degree in electrical engineering at National Sun Yat-sen University. His doctoral dissertation is focused on RF sensing techniques.

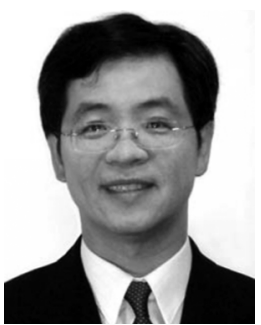

Tzyy-Sheng Horng (S'88-M'92-SM'05) was born in Taichung, Taiwan, on December 7, 1963. He received the B.S.E.E. degree from National Taiwan University, Taipei, Taiwan, in 1985, and the M.S.E.E. and Ph.D. degrees from the University of California at Los Angeles (UCLA), in 1990 and 1992, respectively.

In August 1992, he joined the Department of Electrical Engineering, National Sun Yat-sen University, Kaohsiung, Taiwan, where he is currently a Professor, possessing experience as a Director of the Telecommunication Research and Development Center (2003-2008) and the Institute of Communications Engineering (2004-2007). He has authored or coauthored over 100 technical publications in refereed journals and conferences proceedings. He holds over ten patents. His research interests include RF and microwave integrated circuits and components, RF signal integrity for wireless system-in-package, and digitally assisted RF technologies.

Dr. Horng has served on several Technical Program Committees of international conferences including the IASTED International Conference on Wireless and Optical Communications (WOC), the IEEE Region 10 International Technical Conference (TENCON), the IEEE International Workshop on Electrical Design of Advanced Packaging and Systems (EDAPS), the Asia-Pacific Microwave Conference (APMC), and the IEEE Radio and Wireless Symposium (RWS). He was the recipient of the 1996 Young Scientist Award presented by the International Union of Radio Science and the 1998 Industry-Education Cooperation Award presented by the Ministry of Education, Taiwan.

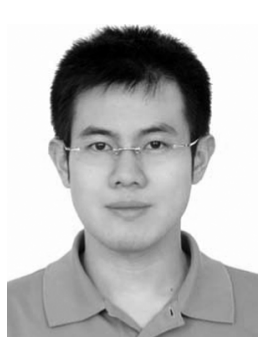

Kang-Chun Peng (S'00-M'05) was born in Taipei, Taiwan, on February 18, 1976. He received the B.S.E.E., M.S.E.E., and Ph.D. degrees from National Sun Yat-sen University, Kaohsiung, Taiwan, in 1998, 2000, and 2005, respectively.

$\mathrm{He}$ is currently an Assistant Professor with the Department of Computer and Communication Engineering, National Kaohsiung First University of Science and Technology, Kaohsiung, Taiwan. His research interests are frequency synthesizers and RF transmitters. 